UG-FT-203/06

CAFPE-73/06

FFUOV-06/09

FTUAM-06/07

hep-th/0606264

October 2006

\title{
The baryon vertex with magnetic flux
}

\author{
Bert Janssen $^{a}$ 1, Yolanda Lozano ${ }^{b}, 2$ and Diego Rodríguez-Gómez ${ }^{c}, 3$ \\ ${ }^{a}$ Departamento de Física Teórica y del Cosmos and \\ Centro Andaluz de Fúsica de Partículas Elementales \\ Universidad de Granada, 18071 Granada, Spain \\ ${ }^{b}$ Departamento de Física, Universidad de Oviedo, \\ Avda. Calvo Sotelo 18, $3300^{\prime}$ Oviedo, Spain \\ ${ }^{c}$ Departamento de Física Teórica $C$-XI, \\ Universidad Autónoma de Madrid, \\ Cantoblanco, 28049 Madrid, Spain
}

\begin{abstract}
In this letter we generalise the baryon vertex configuration of AdS/CFT by adding a suitable instantonic magnetic field on its worldvolume, dissolving D-string charge. A careful analysis of the configuration shows that there is an upper bound on the number of dissolved strings. This could be a manifestation of the stringy exclusion principle. We provide a microscopical description of this configuration in terms of a dielectric effect for the dissolved strings.
\end{abstract}

\footnotetext{
${ }^{1}$ E-mail address: bjanssen@ugr.es

${ }^{2}$ E-mail address: yolanda@string1.ciencias.uniovi.es

${ }^{3}$ E-mail address: Diego.Rodriguez.Gomez@cern.ch
} 


\section{Introduction}

The $A d S / C F T$ duality [1] relates gravity theories in $A d S$ spaces with certain conformal field theories. In particular, it states that IIB string theory on $A d S_{5} \times S^{5}$ is dual to $\mathcal{N}=4 \mathrm{SYM}$ in four dimensions. In the context of the dual field theory, an extremely interesting question is whether the theory is confining or not. In order to study this, the appropriate quantity to look at is the Wilson loop, whose VEV gives the quark-antiquark $(q \bar{q})$ potential. In the case at hand, $\mathcal{N}=4 \mathrm{SYM}$ has no dynamical quarks. However, one can introduce static quarks and compute the appropriate Wilson loop to obtain the $q \bar{q}$ potential. The Wilson loop can be computed both at weak 't Hooft coupling, directly in field theory [2], and at strong 't Hooft coupling, in the gravity side of the correspondence [3, 4]. In both regimes the $q \bar{q}$ potential goes like $1 / d$, where $d$ is the distance between the quark and antiquark. This particular coulombian shape, which exhibits no confinement, is due to the conformal invariance of the theory.

As shown in [1, 4, F-strings ending on the D3-brane and going all the way to the $A d S$ boundary are seen in the dual theory as external quarks or antiquarks, depending on the string's orientation. It is clear then that one can form a $q \bar{q}$ state with a single string coming from the boundary to the D3-brane and then going back to the boundary. Once the coupling to the $S U(N)$ theory is taken into account, the string ends up being U-shaped, with the apex at a distance $u_{0}$ from the stack of D3-branes. This configuration is seen as a $q \bar{q}$ pair on the SYM side, whose energy is computed by means of a rectangular Wilson loop. On the other hand, on the gravity side the energy is computed by minimising the worldsheet area of the string ending on the loop.

From the Wilson loop one can only extract the $q \bar{q}$ potential. However, one would expect that there should be a mechanism to form bound states of $N$ non-dynamical quarks. In [5] precisely this question was asked, namely whether it would also be possible to construct in this set-up a baryon configuration. Roughly speaking, a baryon is a colourless bound state of quarks with finite energy. In the case at hand, where there are no dynamical quarks, it turns out to be possible to construct such a bound state with static external quarks. In [5] the gravitational dual of this bound state of quarks was found in terms of a D5-brane wrapping the $S^{5}$ part of the spacetime geometry. On this D5-brane there are $N$ F-strings attached, stretching from the D5-brane to the boundary of $A d S_{5}$. The endpoints of the $N$ F-strings are then regarded on the dual SYM side as a bound state of $N$ quarks, in other words, as a baryon 4 Indeed it can be shown [5] that the associated wave function satisfies the required symmetry properties.

In this letter we generalise this baryon vertex configuration by adding a new quantum number. The key point is to realise that $S^{5}$ can be seen as an $S^{1}$ bundle over $C P^{2}$. The $S^{1}$ fibre is a non-trivial $U(1)$ gauge bundle on the $C P^{2}$ base, and this allows to switch on a magnetic BI field on the worldvolume of the D5-brane, proportional to the curvature tensor of the fibre connection. As we will see, the effect of this field is to dissolve D1-branes wound

\footnotetext{
${ }^{4}$ Since the quarks are non-dynamical, this represents a mechanism to form the baryon, and is referred to as the baryon vertex.
} 
around the $S^{1}$ direction on the D5-brane.

The interest of this generalised baryon vertex is twofold. On one hand, the analysis of the equations of motion reveals that there is a bound on the number of D-strings that can be dissolved in the D5-brane. This is an interesting phenomenon, which could be related to the stringy exclusion principle [6]. Indeed, by dissolving D-strings in the configuration we are inducing a non-zero winding charge along a cycle of the $S^{5}$, and these winding charges appear in the dual field theory as non-zero charges under certain $U(1)$ subgroups of the $S O(6)$ R-symmetry [7], which are bounded due to conformal invariance. A complete analysis in the field theory context is however beyond the scope of this letter.

On the other hand, the fact that we have dissolved D-strings on the worldvolume of the D5brane hints at the existence of an alternative microscopical description in terms of non-Abelian D-strings polarising due to a dielectric effect [8]. We give such a microscopical description in terms of D1-branes expanding into a fuzzy spherical D5-brane using the action of [8]. We also consider the S-dual of the baryon vertex with magnetic flux, which consists on a spherical NS5-brane with dissolved F-strings, and with N D1-branes attached to it. We show that this configuration can also be described microscopically in terms of F-strings expanding into a fuzzy spherical NS5-brane by dielectric effect.

This letter is organised as follows. In section 2 we present the D5-brane description of the generalised baryon vertex. We start in subsection 2.1 by revisiting the construction of the original baryon vertex as given by [5], and then generalise this construction in subsection 2.2 to include a magnetic BI vector on the worldvolume. In subsection 2.3 we study the dynamics of this configuration and show that there is a bound on the number of dissolved strings. Section 3 is devoted to the microscopic description of the generalised baryon vertex in terms of nonAbelian D-strings. In subsection 3.1 we calculate the energy of the configuration of multiple coinciding D-strings polarising into a fuzzy spherical D5-brane. In subsection 3.2 we show how the $N$ fundamental strings that connect the (dielectric) D5-brane to the gauge theory on the boundary arise in the microscopical set-up. Subsection 3.3 contains the description of the S-dual of the baryon vertex with magnetic flux in terms of fundamental strings expanding into a fuzzy spherical NS5-brane. The action describing coinciding fundamental strings is constructed from the action for coinciding Type IIA gravitational waves of [9] using T-duality. In the conclusions we review the main points of our construction.

\section{The baryon vertex with magnetic flux}

\subsection{The baryon vertex revisited}

We start by reviewing the major points in the construction of the baryon vertex, as given in 5. Consider a probe D5-brane wrapped on the 5 -sphere and static in a fixed point in $\operatorname{AdS}$. In the $A d S_{5} \times S^{5}$ background there is no 6 -form $\mathrm{R}$ - $\mathrm{R}$ potential to which the probe brane can couple, however the presence of the 4 -form R-R field in the Chern-Simons action induces a coupling to the BI field strength $F=d A$ of the form

$$
S_{C S}=-T_{5} \int_{\mathbb{R} \times S^{5}} P\left[C^{(4)}\right] \wedge F
$$


In our specific setting, the only non-zero contribution is that of the coupling of the magnetic part of the R-R form to the electric component of $F$. Integrating by parts, we find that this term can be rewritten as

$$
S_{C S}=T_{5} \int_{\mathbb{R} \times S^{5}} P\left[G^{(5)}\right] \wedge A,
$$

where $G^{(5)}=d C^{(4)}$ is the R-R 5-form field strength. In our particular background, we have that $G^{(5)}=4 L^{4} \sqrt{g_{S^{5}}}$, such that $\int_{S^{5}} G^{(5)}=4 \pi^{2} N$ (in units where $2 \pi l_{s}^{2}=1$ ), with $N$ the number of D3-branes that build up the background. If we therefore take as an Ansatz for the $\mathrm{BI}$ vector

$$
A=A_{t}(t) d t
$$

it is clear that the coupling (2.2) factorises as

$$
S_{C S}=T_{5} \int_{S^{5}} G^{(5)} \int d t A_{t}=N T_{1} \int d t A_{t},
$$

where we have taken into account that the tension of the D5-brane and the tension of a string are related by $4 \pi^{2} T_{5}=T_{1}$. Therefore, one can interpret that the coupling (2.1) is inducing $N$ units of BI electric charge on the D5-brane, such that the total action for the wrapped D5-brane can be written as

$$
S=S_{D B I}+N T_{1} \int d t A_{t}
$$

However we have to check whether the Ansatz (2.3) is consistent with the equations of motion of the D5-brane system (2.5). As (2.3) implies that $F=0$, it is clear that the equation of motion of $A$ is given by

$$
0 \equiv \frac{\partial \mathcal{L}}{\partial A_{t}}=N T_{1}
$$

In other words, the equations of motion imply that the Ansatz (2.3) is only compatible with the action (2.5) if the total BI electric charge on the D5-brane is zero, as it is wrapped on a compact manifold. However, there is a consistent way of inducing a non-zero BI electric charge in the worldvolume of the D5-brane, by cancelling this charge with the charge induced by the endpoints of $N$ open fundamental strings (with appropriate orientation) stretching between the D5-brane and the boundary of the $A d S$ space. The action (2.5) is therefore not describing the entire system, but only the D5-brane part. In order to describe the full dynamics one has to add the action for the open strings, consisting of $N$ copies of the Nambu-Goto action $S_{F 1}$, and a boundary term contribution $T_{1} \int A_{t} d t$ from the endpoints:

$$
S_{\text {total }}=S_{D B I}+N T_{1} \int d t A_{t}+N S_{F 1}-N T_{1} \int d t A_{t}
$$

Note that the contribution from the open string endpoints cancels exactly the Chern-Simons term in the D5-brane action, such that the total system is described by [10]

$$
S_{\text {total }}=S_{D B I}+N S_{F 1}
$$

The configuration that we have just described is the so-called baryon vertex. Since the $N$ F-strings, stretching from the D5-brane all the way to the $A d S$ boundary, have the same 
orientation, the dual configuration on the CFT side corresponds to the bound state of $N$ (anti)quarks, which is gauge invariant and antisymmetric under the interchange of any two quarks [5].

\subsection{Adding magnetic flux to the baryon vertex}

It is well known that $S^{5}$ can be regarded as a $U(1)$ fibre over $C P^{2}$ with a non-trivial fibre connection. From the $C P^{2}$ point of view, the $U(1)$ connection, $B$, can be seen as a non-trivial gauge bundle inducing a non-zero instanton number [11, 12. In view of this, it seems natural to consider a generalisation of the baryon vertex in which magnetic components of the BI field strength are switched on, which are proportional to $d B$.

In the $S^{5}$ fibre coordinates the $A d S_{5} \times S^{5}$ background reads

$$
\begin{aligned}
& d s^{2}=\frac{u^{2}}{L^{2}} \eta_{a b} d x^{a} d x^{b}+\frac{L^{2}}{u^{2}} d u^{2}+L^{2}\left((d \chi-B)^{2}+d s_{C P^{2}}^{2}\right), \\
& C_{a b c d}=L^{-4} u^{4} \epsilon_{a b c d},
\end{aligned}
$$

where $d s_{C P^{2}}^{2}$ stands for the Fubini-Study metric on $C P^{2}, \chi$ is taken along the $U(1)$ fibre and $B$ is the connection of the fibre bundle. Explicitly [13]

$$
\begin{aligned}
& B=-\frac{1}{2} \sin ^{2} \varphi_{1}\left(d \varphi_{4}+\cos \varphi_{2} d \varphi_{3}\right), \\
& d s_{C P^{2}}^{2}=d \varphi_{1}^{2}+\frac{1}{4} \sin ^{2} \varphi_{1}\left(d \varphi_{2}^{2}+\sin ^{2} \varphi_{2} d \varphi_{3}^{2}+\cos ^{2} \varphi_{1}\left(d \varphi_{4}+\cos \varphi_{2} d \varphi_{3}\right)^{2}\right) .
\end{aligned}
$$

The fibre connection $B$ satisfies the following properties [1]

$$
d B={ }^{\star}(d B), \quad \int_{C P^{2}} d B \wedge d B=4 \pi^{2},
$$

where the Hodge star is taken with respect to the metric (2.10) on $C P^{2}$.

In this system of coordinates the baryon vertex consists on the D5-brane wrapped around the $S^{5}$ and the fundamental strings laying in the $u$-direction of $A d S_{5}$ [4]. As mentioned above, besides the electric components of the BI field strength, representing the charges induced by the F-strings ending on the D5-brane, one could think of turning on also magnetic components. Due to the fact that $C P^{2}$ allows instanton solutions, it is natural to take the magnetic components living in the $C P^{2}$ and proportional to the curvature tensor of the $U(1)$ fibre connection $B$,

$$
F=2 n d B \text {. }
$$

With this Ansatz $F$ satisfies the same properties (2.11) as the fibre connection $d B$, namely it is selfdual and

$$
\int_{C P^{2}} F \wedge F=8 \pi^{2} n^{2}
$$

This integral is non-zero because it is the product of two integrals $\oint F$ over non-trivial twocycles in $C P^{2}$. Since $\oint F=2 \pi n$ due to the Dirac quantization condition, $n$ represents 
the winding number of D3-branes wrapped around each of the two-cycles. Note that the winding number must be the same on each cycle in order to preserve the selfduality condition. Moreover, if we want that some of the supersymmetries of the D5-brane, if any, are preserved, the two D3-branes must be wrapped with the same orientation.

With this choice for the BI field strength it is clear that there are no other couplings in the Chern-Simons action besides the ones we already considered in (2.1). The Born-Infeld action however is given by

$$
S_{D B I}=-T_{5} \int d^{6} \xi \frac{u}{L} \sqrt{\operatorname{det}\left(g_{\alpha \beta}+F_{\alpha \beta}\right)}
$$

where the coordinates $x^{\alpha}$ indicate the angles on the $S^{5}$. Since $F$ is selfdual, the determinant under the square root is a perfect square, yielding

$$
S_{D B I}=-T_{5} \int d^{6} \xi u \sqrt{g_{S^{5}}}\left(L^{4}+2 F_{\alpha \beta} F^{\alpha \beta}\right) .
$$

The Ansatz (2.12) is consistent with the action (2.15), as is reflected in the fact that the equations of motion for the magnetic components of $F$ are given by $d F=0$, which is indeed satisfied by (2.12). Finally, substituting the expression for $F$ in the action and integrating over the $S^{5}$ directions we obtain the following expression for the energy of the spherical D5-brane:

$$
E_{D 5}=8 \pi^{3} T_{5} u\left(n^{2}+\frac{L^{4}}{8}\right) .
$$

Note that this energy consists of two parts: one contribution from the tension of the 5-brane wrapped around the five-sphere and one from the magnetic flux of the BI vector.

While the electric components of $F$ induce $N$ units of BI charge on the D5-brane worldvolume through the coupling (2.1), the magnetic components induce a non-zero instanton number $n^{2}$, due to (2.13). In particular, the Chern-Simons coupling

$$
S_{C S}=\frac{1}{2} T_{5} \int_{\mathbb{R} \times S^{5}} P\left[C^{(2)}\right] \wedge F \wedge F
$$

can be integrated directly over the $C P^{2}$ directions, yielding

$$
S_{C S}=n^{2} T_{1} \int_{\mathbb{R} \times S^{1}} P\left[C^{(2)}\right]
$$

where we have used again that $T_{1}=4 \pi^{2} T_{5}$. Even though in $A d S_{5} \times S^{5} C^{(2)}$ is zero, this coupling indicates that the magnetic flux is inducing $n^{2} \mathrm{D}$-string charge in the configuration. These strings are wound around the fibre direction $\chi$.

Note that $n$ D3-brane charge is also induced in the configuration through the Chern-Simons coupling

$$
S_{C S}=-T_{5} \int_{\mathbb{R} \times S^{5}} P\left[C^{(4)}\right] \wedge F,
$$

with the D3-branes wrapped on the non-trivial two-cycles of the $C P^{2}$. However, only the charge at the intersection of the two D3-branes contributes to the energy. In fact, expression 
(2.16) is precisely of the form of a threshold BPS intersection for D1- and D5-branes, being the total energy just the sum of the energies of each of the constituents.

Let us now discuss the influence of the $N$ fundamental strings that stretch from the D5brane to the boundary of $A d S_{5}$. In order to keep the spherical D5-brane undeformed the F-strings must be uniformly scattered over the five-sphere. Otherwise, if a significant number of strings are joined at the same point, their backreaction is not negligible and they will start to deform the 5-sphere [14, 15. Then, in this limit, one would need to consider the full DBI problem, in which the F-strings are seen as a spike in the worldvolume of the D5-brane, in the spirit of [16, 17, 18]. However, taking the $N$ fundamental strings to join the D5-brane in different points breaks all the supersymmetry, since although each string preserves one half, the fact that they take different positions on the D5-brane makes that the preserved Killing spinors of each string are different, such that all supersymmetries are broken [15]. We will see below that this breaking results in the fact that the configuration has a binding energy.

The spherical D5-brane with magnetic flux that we have discussed in this section is very similar to the spherical D2-brane probe with dissolved D0-brane charge of [19]. It was shown in [8] that there exists a complementary, microscopical description of this system in terms of D0-branes expanding by dielectric effect into a fuzzy spherical D2-brane, and that when the number of D0-branes is large enough, the microscopical and D2-brane descriptions coincide. The analogy with our case suggests that there should exist a microscopical description of the baryon vertex with magnetic flux, in terms of multiple non-Abelian D-strings, expanding into a fuzzy spherical D5-brane. We will provide this microscopical description in the next section. In the remaining part of this section we will first analyse the influence of the magnetic flux on the dynamics of the baryon vertex.

\subsection{The bound on the instanton number}

It was argued in [10] that in order to analyse the stability of the baryon vertex in the $u$ direction (i.e. against perturbations in the holographic direction of $A d S$ ), one has to consider the influence of the external F-strings. The energy $E$ of the baryon vertex is then proportional to $N$ times the energy of a $q \bar{q}$ system, which is in turn inversely proportional to the distance $\ell$ between the quarks [3]. As the proportionality constant between $E$ and $\ell$ is negative, the baryon vertex is indeed stable under perturbations in $u$.

In this subsection we will perform the same calculation in [10, but taking into account the effect of the non-zero magnetic flux on the D5-brane.

The action for the baryon vertex with magnetic flux on the worldvolume of the D5-brane is given by

$$
S=S_{D 5}+S_{N F 1}
$$

with $S_{D 5}$ given by minus the time integration of (2.16). On the other hand, the F-strings connecting the D5-brane and a quark on the boundary can be parametrised by the worldvolume coordinates $\{t, x\}$ and the position in $A d S$ by $u=u(x)$. Then, the Nambu-Goto action is given by

$$
S_{N F 1}=-N T_{1} \int d t d x \sqrt{\left(u^{\prime}\right)^{2}+\frac{u^{4}}{L^{4}}}
$$


where $u^{\prime}$ denotes the derivative of $u(x)$ with respect to $x$. Following the analysis of [10], the equations of motion associated to the system come in two sets: the bulk equation of motion for the strings, and the boundary equation of motion (as we are dealing with open strings), which contains as well a term coming from the D5-brane. One can show easily that these equations of motion are:

$$
\begin{aligned}
& \frac{u^{4}}{\sqrt{\left(u^{\prime}\right)^{2}+\frac{u^{4}}{L^{4}}}}=\text { const, } \\
& \frac{u_{0}^{\prime}}{\sqrt{\left(u_{0}^{\prime}\right)^{2}+\frac{u_{0}^{4}}{L^{4}}}}=\frac{\pi L^{4}}{4 N}\left(1+\frac{8 n^{2}}{L^{4}}\right),
\end{aligned}
$$

for the bulk and the boundary respectively, with $u_{0}$ the position of the baryon vertex in the holographic direction and $u_{0}^{\prime}=u^{\prime}\left(u_{0}\right)$. For future convenience, let us call

$$
\sqrt{1-\beta^{2}}=\frac{\pi L^{4}}{4 N}\left(1+\frac{8 n^{2}}{L^{4}}\right)
$$

Notice that in our conventions, $g_{s}=1$ and $2 \pi l_{s}^{2}=1$, we have that

$$
L^{4}=4 \pi g_{s} l_{s}^{4} N=\frac{N}{\pi},
$$

and we can rewrite (2.24) as

$$
\beta^{2}=1-\frac{1}{16}\left(1+\frac{8 \pi n^{2}}{N}\right)^{2}
$$

Equations (2.22) and (2.23) can then be combined into a single one,

$$
\frac{u^{4}}{\sqrt{\left(u^{\prime}\right)^{2}+\frac{u^{4}}{L^{4}}}}=\beta u_{0}^{2} L^{2} .
$$

In the absence of magnetic BI flux on the worldvolume, $\beta=\sqrt{15 / 16}$, as in $[10]$. However, in general for non-zero $n^{2}$, we have to make sure that $\beta$ is real (as $u$ is real), which from (2.26) implies that

$$
\frac{n^{2}}{N} \leq \frac{3}{8 \pi}
$$

Surprisingly, we find that there is a bound on the number of D-strings that can be dissolved in the configuration, which depends on the number of D3-branes that source the background.

Integrating the equation of motion, we find that the size of the baryon $\ell$ is given by

$$
\ell=\frac{L^{2}}{u_{0}} \int_{1}^{\infty} d y \frac{\beta}{y^{2} \sqrt{y^{4}-\beta^{2}}}
$$

with $y=u / u_{0}$. This integral can be solved in terms of hypergeometric functions [10]. In Figure 1 we have plotted the radius $\ell$ of the baryon as a function of $\frac{n^{2}}{N}$. The plot reveals that 


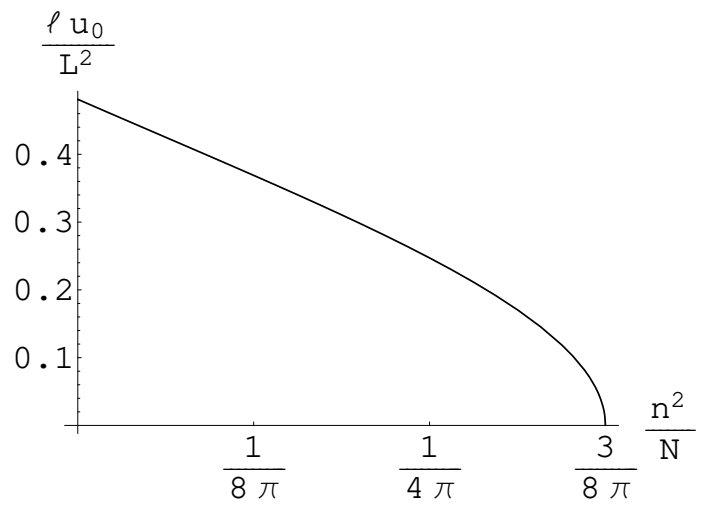

Figure 1: Radius of the baryon (in units of $L^{2} / u_{0}$ ) as a function of $\frac{n^{2}}{N}$.

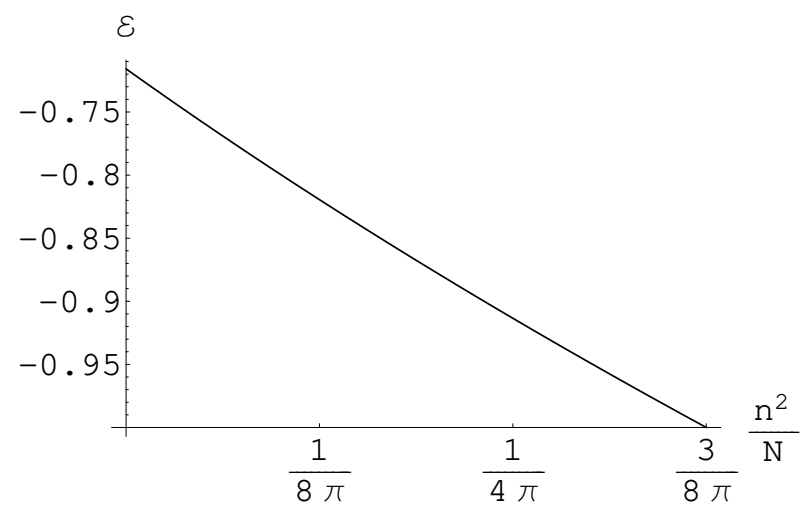

Figure 2: The energy (in units of $u_{0}$ ) of the baryon vertex as a function of $\frac{n^{2}}{N}$.

the radius of the baryon cannot be continued outside the allowed domain given by (2.28). Note that the size of the baryon vertex goes to zero as we saturate the bound.

Finally, the energy for a single string is, in terms of $u_{0}$ :

$$
E=T_{1} u_{0}\left\{\int_{1}^{\infty} d y\left[\frac{y^{2}}{\sqrt{y^{4}-\beta^{2}}}-1\right]-1\right\}
$$

Notice that this expression has the same form than the expression in [10], and indeed takes the same value for $n=0$. In particular, the dependence on $\sqrt{g^{2} N}$ and on $u_{0}$ is unaltered, as expected by conformal invariance. The explicit dependence of the energy on the ratio $\frac{n^{2}}{N}$ can be seen in Figure 2. As expected, the configuration is only well defined for $\frac{n^{2}}{N}$ inside the allowed interval.

The fact that we find a bound on the number of dissolved D1-branes due to the dynamics of the F-strings, is quite surprising, and it is not entirely clear to us what its interpretation is. For a brief discussion we refer to the conclusions. 


\section{The microscopic description of the baryon vertex}

As we mentioned at the end of subsection 2.2, the fact that the magnetic flux in the D5brane worldvolume induces D1-brane charge, suggests a close analogy with the dielectric effect described in [19, 8]. In this section we will show that it is indeed possible to give an alternative, microscopic description of the baryon vertex, in terms of a fuzzy spherical D5-brane built up out of dielectrically expanded D1-branes.

\subsection{D1-branes polarising to a 5-brane}

The action describing the dynamics of $n^{2}$ coinciding D1-branes is the non-Abelian action given in [8], which for the $A d S_{5} \times S^{5}$ background reduces to the form

$$
\begin{aligned}
S_{n^{2} D 1}= & -T_{1} \int d^{2} \xi \operatorname{STr}\left\{\sqrt{\left|\operatorname{det}\left(P\left[g_{\mu \nu}+g_{\mu i}\left(Q^{-1}-\delta\right)^{i}{ }_{j} g^{j k} g_{k \nu}\right]\right) \operatorname{det} Q\right|}\right\} \\
& +T_{1} \int d^{2} \xi \operatorname{STr}\left\{P\left[i\left(\mathrm{i}_{X} \mathrm{i}_{X}\right) C^{(4)}-\frac{1}{2}\left(\mathrm{i}_{X} \mathrm{i}_{X}\right)^{2} C^{(4)} \wedge \mathcal{F}\right\},\right.
\end{aligned}
$$

where $g_{\mu \nu}$ is the metric in $A d S_{5} \times S^{5}$ and

$$
\begin{aligned}
& Q^{i}{ }_{j}=\delta_{j}^{i}+i\left[X^{i}, X^{k}\right] g_{k j}, \\
& \left(\left(\mathrm{i}_{X} \mathrm{i}_{X}\right) C^{(4)}\right)_{\mu \nu}=\frac{1}{2}\left[X^{\lambda}, X^{\rho}\right] C_{\rho \lambda \mu \nu}^{(4)}, \\
& \left(\mathrm{i}_{X} \mathrm{i}_{X}\right)^{2} C^{(4)}=\frac{1}{4}\left[X^{\lambda}, X^{\rho}\right]\left[X^{\nu}, X^{\mu}\right] C_{\mu \nu \rho \lambda}^{(4)} .
\end{aligned}
$$

Inspired by the coupling (2.18) in the D5-brane calculation we wind the D-strings around the $U(1)$ fibre direction $\chi$ and let them expand into the $C P^{2}$. In this way we obtain a fuzzy version of the $S^{5}$ as an Abelian $U(1)$ fibre over a fuzzy $C P^{2}$, similar to the microscopic description of 5-dimensional giant gravitons in $A d S_{4} \times S^{7}$ and $A d S_{7} \times S^{4}$ found in [20].

In the $A d S_{5} \times S^{5}$ background the Chern-Simons couplings in (3.1) vanish. Therefore, the expansion of the strings into a fuzzy $C P^{2}$ is caused by the couplings in the Born-Infeld part of the action and, thus, it is entirely due to a gravitational dielectric effect, analogous to the configurations described in [21].

A fuzzy version of $C P^{2}$ is well known (see for example [22]). $C P^{2}$ is the coset manifold $S U(3) / U(2)$, and can be defined as the submanifold of $\mathbb{R}^{8}$ determined by the constraints

$$
\sum_{i=1}^{8} x^{i} x^{i}=1, \quad \sum_{j, k=1}^{8} d^{i j k} x^{j} x^{k}=\frac{1}{\sqrt{3}} x^{i},
$$

where $d^{i j k}$ are the components of the totally symmetric $S U(3)$-invariant tensor. In our coor- 
dinates (2.10]) we have (see [20])

$$
\begin{array}{rlrl}
x^{1} & =\frac{\sqrt{3}}{2} \sin 2 \varphi_{1} \cos \frac{\varphi_{2}}{2} \cos \frac{\varphi_{4}+\varphi_{3}}{2}, & x^{5} & =-\frac{\sqrt{3}}{2} \sin 2 \varphi_{1} \sin \frac{\varphi_{2}}{2} \sin \frac{\varphi_{4}-\varphi_{3}}{2}, \\
x^{2} & =-\frac{\sqrt{3}}{2} \sin 2 \varphi_{1} \cos \frac{\varphi_{2}}{2} \sin \frac{\varphi_{4}+\varphi_{3}}{2}, & x^{6}=\frac{\sqrt{3}}{2} \sin ^{2} \varphi_{1} \sin \varphi_{2} \cos \varphi_{3}, \\
x^{3}=\frac{\sqrt{3}}{2}\left[\sin ^{2} \varphi_{1}\left(1+\cos ^{2} \frac{\varphi_{2}}{2}\right)-1\right], & x^{7}=-\frac{\sqrt{3}}{2} \sin ^{2} \varphi_{1} \sin \varphi_{2} \sin \varphi_{3}, \\
x^{4}=\frac{\sqrt{3}}{2} \sin 2 \varphi_{1} \sin \frac{\varphi_{2}}{2} \cos \frac{\varphi_{4}-\varphi_{3}}{2}, & x^{8}=\frac{1}{2}\left(3 \sin ^{2} \varphi_{1} \sin ^{2} \frac{\varphi_{2}}{2}-1\right),
\end{array}
$$

for which $\frac{1}{3} \sum_{i=1}^{8}\left(d x^{i}\right)^{2}=d s_{C P^{2}}^{2}$ is the Fubini-Study metric (2.10). A fuzzy version of $C P^{2}$ can be obtained by imposing the conditions (3.3) at the level of matrices. Define a set of coordinates $X^{i}(i=1, \ldots, 8)$ as

$$
X^{i}=\frac{T^{i}}{\sqrt{\left(2 n^{2}-2\right) / 3}},
$$

with $T^{i}$ the generators of $S U(3)$ in the $n^{2}$-dimensional irreducible representations $(k, 0)$ or $(0, k)$, with $n^{2}=(k+1)(k+2) / 2$ (see [20, 22] for more details). Nothe that $\left(2 n^{2}-2\right) / 3$ is the quadratic Casimir of $S U(3)$ in these representations. The first constraint in (3.3) is then trivially satisfied through the quadratic Casimir of the group, whereas the rest of the constraints are satisfied for any $n^{2}$. The commutation relations between the $X^{i}$ are given by

$$
\left[X^{i}, X^{j}\right]=\frac{i f^{i j k}}{\sqrt{\left(2 n^{2}-2\right) / 3}} X^{k},
$$

with $f^{i j k}$ the structure constant of $S U(3)$ in the algebra of the Gell-Mann matrices $\left[\lambda^{i}, \lambda^{j}\right]=$ $2 i f^{i j k} \lambda^{k}$.

Substituting the non-commutative Ansatz above in the action (3.1) and particularising to the $A d S_{5} \times S^{5}$ background, we find

$$
\begin{aligned}
S_{n^{2} D 1} & =-T_{1} \int d t d \chi u \operatorname{STr}\left\{\mathbb{1}+\frac{L^{4}}{4\left(2 n^{2}-2\right)} \mathbb{1}\right\} \\
& =-2 \pi n^{2} T_{1} \int d t u\left(1+\frac{L^{4}}{8\left(n^{2}-1\right)}\right),
\end{aligned}
$$

since, remarkably,

$$
\operatorname{det} Q=\left(\mathbb{1}+\frac{L^{4}}{4\left(2 n^{2}-2\right)} \mathbb{1}\right)^{2},
$$

in the large $n$ limit5. It is to be emphasized that the fact that the $\operatorname{det} Q$ is a perfect square is the microscopical analogous of the perfect square that we obtained for the DBI action in the macroscopic case.

The energy of the $n^{2}$ expanded D1-branes is then given by

$$
E_{n^{2} D 1}=2 \pi u T_{1}\left(n^{2}+\frac{n^{2} L^{4}}{8\left(n^{2}-1\right)}\right) .
$$

\footnotetext{
${ }^{5}$ To be more precise, we are neglecting higher order powers of $L^{2} / n$. This is the right limit to study the matching with the macroscopical description of the previous section.
} 
Taking into account that the tensions of the D1- and the D5-brane are related by $T_{1}=4 \pi^{2} T_{5}$, it is easy to see that in the limit where the number of D1-branes $n^{2} \rightarrow \infty$, the above expression reduces to the energy of the macroscopic D5-brane, given by (2.16).

\subsection{The $N$ F-strings in the microscopic description}

So far we have compared the energy of the spherical D5-brane of the baryon vertex to the energy of the configuration built up by $n^{2}$ D1-branes expanding into a D5-brane with the topology of a fuzzy 5-sphere. We have shown that the two descriptions agree in the limit where the instanton number on the D5-brane is very large. However an essential part in the construction of the baryon vertex are the $N$ fundamental strings that stretch from the D5brane in the interior to the boundary of $A d S_{5}$. In this subsection we show how these strings arise in the microscopical setup.

The CS action for coincident D-strings contains the following couplings to the $C^{(4)} \mathrm{R}-\mathrm{R}$ potential:

$$
S_{C S}=T_{1} \int d t d \chi \operatorname{STr}\left\{P\left[\left(\mathrm{i}_{X} \mathrm{i}_{X}\right) C^{(4)}\right]-P\left[\left(\mathrm{i}_{X} \mathrm{i}_{X}\right)^{2} C^{(4)}\right] \wedge \mathcal{F}\right\}
$$

where $\mathcal{F}=d \mathcal{A}+[\mathcal{A}, \mathcal{A}]$ is the $U\left(n^{2}\right)$ BI field strength.

The first term in (3.10) is zero in the $A d S_{5} \times S^{5}$ background. The second term, in turn, can be written as

$$
S_{C S}=\frac{T_{1}}{4} \int d t d \chi \operatorname{STr}\left\{\left[X^{i}, X^{j}\right]\left[X^{k}, X^{l}\right] C_{i j k l}^{(4)} \partial_{\chi} \mathcal{A}_{t}\right\},
$$

in the gauge $\mathcal{A}_{\chi}=0$. Integrating by parts we have that

$$
S_{C S}=-\frac{T_{1}}{4} \int d t d \chi \operatorname{STr}\left\{\left[X^{i}, X^{j}\right]\left[X^{k}, X^{l}\right] G_{\chi i j k l}^{(5)} \mathcal{A}_{t}\right\}
$$

Taking into account that in the non-commutative coordinates introduced in (3.5), $G^{(5)}$ is given by [20]

$$
G_{\chi i j k l}^{(5)}=L^{4} f_{[i j}^{m} f_{k l]}^{n} X^{m} X^{n}
$$

we find that

$$
S_{C S}=\frac{L^{4} T_{1}}{2\left(n^{2}-1\right)} \int d t d \chi \operatorname{STr}\left\{\mathcal{A}_{t}\right\}
$$

where we have made use of the commutation relations (3.6). In analogy with the Abelian case (2.3), we can take as an Ansatz for $\mathcal{A}$,

$$
\mathcal{A}=A_{t}(t) \mathbb{1} d t
$$

Integrating over $\chi$ and taking into account that $L^{4}=N / \pi$ we find finally that

$$
S_{C S}=\frac{n^{2}}{n^{2}-1} N T_{1} \int d t A_{t} .
$$


The coupling (3.11) is therefore inducing, in the large $n^{2}$ limit, $N$ BI charges in the configuration 6 These charges have to be cancelled by $N$ fundamental strings ending on the D1-brane system. The dielectric coupling to $C^{(4)}$ in (3.10) will then take care that these strings are expanded over the full $S^{5}$.

We can therefore conclude that our microscopical picture, consisting of multiple coinciding D-strings expanding into a fuzzy D5-brane, reproduces in the large $n^{2}$ limit all the relevant features of the baryon vertex with magnetic flux. Not only did we obtain the same expression for the energy of the D5-brane, but we also found traces of the presence of the fundamental strings stretched between the (dielectric) 5-brane and the boundary of $A d S$.

\subsection{F-strings polarising to a NS5-brane}

Due to the S-duality invariance of the $A d S_{5} \times S^{5}$ background, the baryon vertex with magnetic flux, described in the previous sections, can alternatively be realised as a NS5-brane wrapped on the $S^{5}$, with $N$ D1-branes stretching between the brane and the boundary of the $A d S$ space and with $n^{2}$ F-string charge dissolved in its worldvolume. Microscopically this configuration is described in terms of fundamental strings expanding into a fuzzy NS5-brane. In this subsection we give the details of this description.

An action describing coincident F-strings in Type IIB can be constructed from the action for coincident gravitational waves in Type IIA, using T-duality. Such an action was constructed in [25] to the linearised level in the background fields, and turned out to be the S-dual of the action for coincident D1-branes of [8], linearised in the background fields. Since in this picture the dynamics of the non-Abelian F-strings is induced by the open D-strings that end on them, this action is adequate to describe the system in the strong coupling regime.

However, given the S-duality invariance of the $A d S_{5} \times S^{5}$ background, the non-Abelian action for F-strings can well be used here. We will start by constructing an action valid beyond the linearised level, and therefore suitable for the study of the $A d S_{5} \times S^{5}$ background.

Using the action of [26, 9] for coincident Type IIA gravitational waves, valid to all orders in the background fields, we can construct an action describing coincident Type IIB F-strings by T-dualising along the direction of propagation of the waves.

The action for coincident Type IIA gravitational waves contains a worldvolume scalar field associated to D0-branes "ending" on the system (see [26]). We will set to zero this field for simplicity and take as well $B^{(2)}=C^{(1)}=0$. This is suitable for the study of the $A d S_{5} \times S^{5}$ background. We then have (see [26, 9])

$$
\begin{aligned}
S_{n^{2} W_{A}} & =-T_{W} \int d \tau \operatorname{STr}\left\{k^{-1} \sqrt{\left|\operatorname{det}\left(P\left[E_{\mu \nu}+E_{\mu i}\left(Q^{-1}-\delta\right)^{i}{ }_{j} E^{j k} E_{k \nu}\right]\right) \operatorname{det} Q\right|}\right\} \\
+ & T_{W} \int d \tau \operatorname{STr}\left\{-P\left[k^{-1} k^{(1)}\right]+i P\left[\left(\mathrm{i}_{X} \mathrm{i}_{X}\right) C^{(3)}\right]+\frac{1}{2} P\left[\left(\mathrm{i}_{X} \mathrm{i}_{X}\right)^{2} \mathrm{i}_{k} B^{(6)}\right]\right\}
\end{aligned}
$$

\footnotetext{
${ }^{6}$ The fact that the number of strings is $N$ only in the large $n^{2}$ limit is similar to the construction of the fuzzy funnels of 23, 24], where D3- and D5-branes are shown to have integer charges only in the limit of infinite D1-branes.
} 
where

$$
\begin{aligned}
E_{\mu \nu} & =g_{\mu \nu}-k^{-2} k_{\mu} k_{\nu}+k^{-1} e^{\phi}\left(i_{k} C^{(3)}\right)_{\mu \nu} \\
Q_{j}^{i} & =\delta_{j}^{i}+i e^{-\phi} k\left[X^{i}, X^{k}\right] E_{k j} .
\end{aligned}
$$

Here $k^{\mu}$ is a Killing vector pointing on the direction of propagation of the gravitational waves 7 $B^{(6)}$ is the NS-NS 6-form potential. Note that (3.17) is a gauged sigma model, in which the Killing direction does not appear as a physical degree of freedom [27.

T-dualising the above action along the Killing direction, we get a non-Abelian action for $n^{2}$ F-strings in Type IIB:

$$
\begin{aligned}
S_{n^{2} F 1}=- & T_{1} \int d \tau d \sigma \mathrm{S} \operatorname{Tr}\left\{\sqrt{\left|\operatorname{det}\left(P\left[E_{\mu \nu}+E_{\mu i}\left(Q^{-1}-\delta\right)^{i}{ }_{j} E^{j k} E_{k \nu}\right]\right) \operatorname{det} Q\right|}\right\} \\
& -T_{1} \int d \tau d \sigma \operatorname{STr}\left\{P\left[B^{(2)}\right]+i P\left[\left(\mathrm{i}_{X} \mathrm{i}_{X}\right) C^{(4)}\right]-\frac{1}{2} P\left[\left(\mathrm{i}_{X} \mathrm{i}_{X}\right)^{2} B^{(6)}\right]\right\},
\end{aligned}
$$

where now

$$
\begin{aligned}
E_{\mu \nu} & =g_{\mu \nu}+e^{\phi} C_{\mu \nu}^{(2)}, \\
Q^{i}{ }_{j} & =\delta_{j}^{i}+i e^{-\phi}\left[X^{i}, X^{k}\right] E_{k j} .
\end{aligned}
$$

This action is no longer a gauged sigma model, as it can be written in a completely covariant way. Although some of the fields are set to zero due to the truncation in (3.17) we see that (3.19) is just the S-dual of the action for $n^{2}$ coincident D-strings of [8]. In particular, the nonAbelian worldvolume scalar associated to D0-branes ending on the Type IIA waves is mapped under T-duality into a non-Abelian vector field which is now associated to D1-branes ending on the system of fundamental strings. One can check at the linearised level (see [25]) that the field strength of this vector field appears in the action for the F-strings exactly as predicted by S-duality.

Using the action (3.19) to describe $n^{2}$ F-strings in the $A d S_{5} \times S^{5}$ background is now straightforward. The computation of the energy of the baryon vertex reduces to the same computation of subsection 3.1. However the strings expand now into a fuzzy NS5-brane, since the configuration acts as a source for the $B^{(6)}$ potential through the last coupling in (3.19). The energy of the configuration is given by

$$
E_{n^{2} F 1}=2 \pi u T_{1}\left(n^{2}+\frac{n^{2} L^{4}}{8\left(n^{2}-1\right)}\right),
$$

which matches exactly the result (3.9) obtained from the D1-brane calculation.

Finally, the S-dual of the coupling (3.11) shows that $N$ open D1-branes must be added to the configuration stretching between the NS5-brane and the boundary of the AdS space. Therefore we have provided a microscopical description of the (generalised) baryon vertex in terms of a spherical NS5-brane with $N$ D1-branes attached to it 5 .

\footnotetext{
${ }^{7}$ In our notation $k^{(1)}=g_{\mu \nu} k^{\nu} d x^{\mu}$. The coupling to $k^{(1)}$ in 3.17) shows that the waves carry momentum along the Killing direction. See [26] for more details.
} 


\section{Conclusions}

The baryon vertex consists on a single probe D5-brane wrapping the $S^{5}$ in $A d S_{5} \times S^{5}$ to which $N$ fundamental strings are attached, running from the D5-brane to the $A d S$ boundary. Since all the strings have the same orientation, this represents a gauge invariant bound state of $N$ quarks, i.e. a baryon [5]. Due to the S-duality invariance of the $A d S_{5} \times S^{5}$ background the baryon vertex can alternatively be realised in terms of a NS5-brane with $N$ D1-branes attached or as a $(p, q)$ 5-brane with $(p, q)$ strings attached.

In this letter, we have found a generalised version of the baryon vertex by writing the $S^{5}$ as an $S^{1}$ fibre bundle over $C P^{2}$. Since $C P^{2}$ admits an instantonic magnetic field proportional to the curvature tensor of the fibre connection, it is possible to consistently plug in a magnetic field on the worldvolume of the D5-brane. These instantons have the effect of dissolving a number $n^{2}$ of D-strings on the D5-brane, wound in the fibre direction. This charge is, however, not topological, since the 5 -sphere has no non-trivial cycles. Therefore, it might be that the baryon vertex with flux represents only a metastable configuration 8 (see fro example [29, 30, 31] for a recent discussion on this type of configurations).

On the other hand, the fact that one can consistently add a number of dissolved D1-branes to the worldvolume of the D5, hints to the existence of an alternative description of the baryon vertex, in terms of expanded D1-branes. We have explicitly provided such a microscopical description of the generalised baryon vertex in terms of D-strings (F-strings) expanding into a D5-brane (NS5-brane) due to Myers dielectric effect. Here the dielectric effect is purely gravitational, i.e. caused by the curvature of the background. Indeed, the CS coupling, as in the macroscopical case, is only indicating the need to introduce the $N$ external F-strings (D-strings) that build up the vertex. The expanding strings are wound along the $S^{1}$ fibre of $S^{5}$ and expand into a fuzzy version of $C P^{2}$. The fuzzy $S^{5}$ is then realised as an Abelian $U(1)$ fibre over a fuzzy $C P^{2}$.

Our construction needs, implicitly, that the F-strings are uniformly scattered over the D5-brane, in such a way that their backreactions are compensated and the D5-brane remains approximately spherical. This however has the effect of breaking all the supersymmetries 15. If we insist in preserving some of the supersymmetries, we have to let all strings end at the same point of the D5-brane, which in turn invalidates our probe approximation. Indeed, in such a case one should look for a full description of the baryonic brane [14, 15], in terms of a single D5-brane developing a spike representing the F-strings [16]-[18, analogous to the D3-brane spike of [28. However, while in that case the binding energy of the configuration is zero, reflecting the fact that it is supersymmetric, this is not the case for our configuration, for which we obtain a non-zero binding energy.

One of the most surprising conclusions of the analysis of the dynamics of the generalised baryon vertex is the fact that the number of dissolved D-strings is bounded from above. A careful study of the baryon vertex along the lines in [10], shows that the configuration is stable against fluctuations in the $u$ direction. In particular, the configuration has the same dependence of the energy on $u_{0}$ as the original vertex. However, in our case the number of

\footnotetext{
${ }^{8}$ We thank the referee for a useful discussion about this point.
} 
dissolved strings must not violate certain bounds imposed by the dynamics of the system. It is likely that this bound is related to the stringy exclusion principle of [6]. Our configuration with non-zero magnetic flux carries a non-zero winding number in the fibre direction of the $S^{5}$, which in terms of the dual field theory will manifest itself as a charge under a specific $U(1)$ subgroup of the $S U(3)$ R-symmetry group [7]. As these charges are bounded due to conformal invariance, one expects to find a bound on the magnetic flux. This is quite similar to the giant graviton effect. Indeed, in $A d S_{5} \times S^{5}$ there exists a giant graviton, which consists of a D3-brane wrapped on an $S^{3}$ inside the $C P^{2}$ part of the $S^{5}$ and moving along the fibre direction. This giant graviton state corresponds in the dual field theory to a chiral primary operator with the same $U(1)$-charge as our configuration. It is surprising however that we can only find the bound on the magnetic flux when the whole system of wrapped D5-branes and F-strings is considered. Another interesting observation is made in [32, where it was noted that in $A d S_{p} \times S^{q}$ spaces the radius $L$ of the $S^{q}$ can be expressed in terms of the dimension $n$ of the representation as $L^{q-1}=l_{P L}^{q-1} n$, if one tries to describe this $q$-sphere as a fuzzy manifold. We leave the precise interpretation of this charge to future investigations.

\section{Acknowledgements}

We wish to thank J.L.F. Barbón, C. Hoyos, J. Hirn, P. Meessen, S. Montero, T. Ortín, J.P. Resco, V. Sanz and especially C. Nuñez and A.V. Ramallo for useful discussions. The work of B.J. is done as part of the program "Ramón y Cajal" of the M.E.C. (Spain). He was also partially supported by the M.E.C. under contract FIS 2004-06823 and by the Junta de Andalucía group FQM 101. The work of Y.L. has been partially supported by CICYT grant BFM2003-00313 (Spain) and by the European Commission FP6 program MRTN-CT-2004005104, in which she is associated to Universidad Autónoma de Madrid. D.R.G. would like to thank the Departamento de Física Teórica y del Cosmos of the Universidad de Granada

for its hospitality while part of this work was done, and for its financial support through a visiting professor grant. He is also grateful to the Departamento de Física de Partículas of the Universitad de Santiago de Compostela for their hospitality in the final stages of this work.

\section{References}

[1] J. Maldacena, Adv. Theor. Math. Phys. 2 (1998) 231, hep-th/9711200.

[2] J. Erickson, G. Semenoff, K. Zarembo, Nucl. Phys. B582 (2000) 155, hep-th/0003055.

[3] J. Maldacena, Phys. Rev. Lett. 80 (1998) 4859, hep-th/9803002.

[4] S. Rey, J. Yee, Eur. Phys. J. C22 (2001) 379, hep-th/9803001.

[5] E. Witten, JHEP 9807 (1998) 006, hep-th/9805112.

[6] J. Maldacena, A. Strominger, JHEP 9812 (1998) 005, hep-th/9804085.

[7] D. Berenstein, C.P. Herzog, I.R. Klebanov, JHEP 0206 (2002) 047, hep-th/0202150.

[8] R. Myers, JHEP 9912 (1999) 022, hep-th/9910053. 
[9] B. Janssen, Y. Lozano, Nucl. Phys. B658 (2003) 281, hep-th/0207199.

[10] A. Brandhuber, N. Itzhaki, J. Sonnenschein, S. Yankielowicz, JHEP 9807 (1998) 020, hep-th/9806158.

[11] A. Trautman, Int. J. Theor. Phys. 16 (1977) 561.

[12] S.W. Hawking, C.N. Pope, Phys. Lett. B73 (1978) 42.

[13] C.N. Pope, Phys. Lett. B97 (1980) 417.

[14] Y. Imamura, Prog. Theor. Phys. 100 (1998) 1263 hep-th/9806162.

[15] Y. Imamura, Nucl. Phys. B537 (1999) 184, hep-th/9807179.

[16] C. Callan, A. Guijosa, K. Savvidy, Nucl. Phys. B547 (1999) 127, hep-th/9810092.

[17] B. Craps, J. Gomis, D. Mateos, A. Van Proeyen, JHEP 9904 (1999) 004, hep-th/9901060.

[18] J. Gomis, A. Ramallo, J. Simón, P. Townsend, JHEP 9911 (1999) 019, hep-th/9907022.

[19] R. Emparan, Phys. Lett. B423 (1998) 71, hep-th/9711106.

[20] B. Janssen, Y. Lozano, D. Rodríguez-Gómez, Nucl. Phys. B712 (2005) 371, hep-th/0411181.

[21] D. Rodríguez-Gómez, JHEP 0601 (2006) 079, hep-th/0509228.

[22] G. Alexanian, A.P. Balachandran, G. Immirzi, B. Ydri, J. Geom. Phys. 42 (2002) 28, hep-th/0103023.

[23] N. Constable, R. Myers, Ø. Tafjord, Phys. Rev. D61 (2000) 106009, hep-th/9911136.

[24] N. Constable, R. Myers, Ø. Tafjord, JHEP 0106 (2001) 023, hep-th/0102080.

[25] D. Brecher, B. Janssen, Y. Lozano, Nucl. Phys. B634 (2002) 23, hep-th/0112180.

[26] B. Janssen, Y. Lozano, Nucl. Phys. B643 (2002) 399, hep-th/0205254.

[27] E. Bergshoeff, B. Janssen, T. Ortín, Phys. Lett. B410 (1997) 131, hep-th/9706117.

[28] C. Callan, J. Maldacena, Nucl. Phys. B513 (1998) 198, hep-th/9708147.

[29] S. Kachru, J. Pearson, H. Verlinde, JHEP 0206 (2002) 021, hep-th/0112197.

[30] K. Intriligator, N. Seiberg, D. Shih, JHEP 0604 (2006) 021, hep-th/0602239.

[31] R. Argurio, M. Bertolini, S. Franco, S. Kachru, Gauge/gravity duality and meta-stable dynamical supersymmetry breaking, hep-th/9610212.

[32] M.M. Sheikh-Jabbari, Inherent Holography in Fuzzy Spaces and an N-tropic Approach to the Cosmological Constant Problem, hep-th/0605110. 